\title{
Enhancement of Voltage Stability by Using SVC for 30-Bus Power System
}

\author{
Dara H. Amin Mohammed \\ Communication Department \\ Technical Institute of Sulaimani \\ Sulaimani Polytechnic University \\ Sulaimani, Iraq \\ dara.amin@spu.edu.iq
}

Volume 4 - Issue 2 |

December 2019

DOI:

10.24017/science.2019.2.14

Received:

15 September 2019

Accepted:

21 November 2019

\section{Abstract}

Voltage stability refers to maintaining the value of the voltage in all busses of the electric network at a steady level (initial operating point) during any sudden disturbance. Voltage instability may happen due to an increase in the demand of the load or in case of any change in the reactive power, thus, the system will goes into uncontrollable and unstoppable decline in the voltage level. The effect of Static Var Compensator (SVC) on voltage stability is discussed in the paper, as well as the improvement of the voltage profile. Usually, SVC and FACTS devices were used for enhancing the voltage level profile and so the stability. Choosing the optimal location for the FACTS devices is essential due to its expensive costs. This paper used sensitivity factor to helpful to determine the most correct placement of FACTS devices in the system. Simulations are performed on Kurdistan Region 30-bus Power System using MATLABPSAT tool. As a result, the voltage of all 30 buses calculated. Based on the "voltage sensitivity factor", the nominated weak buses has been marked which are suitable for placing the FACTS devices in order to improve the limits of the voltage stability of the system. Moreover, depending on the obtained optimal locations, a full analysis of the voltage and powers for the system has applied in two cases, before and after placing SVC respectively which is result in notable stability improvement and losses reduction.

Keywords: Voltage collapse, Voltage Stability Improvement, FACTS, SVC, PSAT simulation, Kurdistan region Power System. 


\section{INTRODUCTION}

Among the emerging technologies in all over the world, voltage stability problems become a notable issue. Voltage collapse phenomenon and overall system breakdown may be happen as a result of a sudden increase load demand or the existence of a non-linearity load [1]

Monirul Islam eta al. [2] have proposed a mitigation strategies to improve the effect of dynamic voltage stability. They considered the issues and affects resultant from the photovoltaic units on the distribution network and how it is affect the network when unbalanced penetration of these units is applied to the network. The authors developed dynamic models of a single phase photovoltaic units. They conclude that whenever the degree of imbalance increased, the level of the instability of the voltage will increase. According to that, modified IEEE 4 bus distribution system was used as a case study to investigate the effect of the proposed strategies. One of the proposed strategies is to let photovoltaic inverters inject a reactive power to the network. The strategy proved that its effectiveness to enhance the dynamic voltage stability. Other scenarios are applied, where in all scenarios the transient voltage severity index has been evaluated.

Mengqi Yao and his colleagues [3] have measure the smallest singular value of the power flow in order to improve the steady state stability of the voltage. They used demand responsive loads to build a multi period approach based an optimum power flow. Their proposed approach is different than the old techniques, in which they kept the total load steady to prevent the system from any frequency change by using the smallest singular value method to control individual load in the network. The worked on developing an intelligent program that uses singular value sensitivities to find optimal AC feasible solutions. The results of testing the developed program on two different IEEE standard system reveal that the voltage stability has been improved depending on the demand response actions. Finally, the researchers proved that their developed program is working six times faster when applied on IEEE 9-bus system. Comparing the developed program with a transitional iterative nonlinear type also has been performed to prove the performance of the developed one.

When an increase in load demand or a change in the condition of the system occurs, or any disturbance in the load demand subject to the system, there is a chance for the system to goes into instability state, which leads to uncontrollable continues or sometimes progressive decrement in the voltage [4]. In general, the uncontrollable continues or progressive decrement in the voltage at some buses will definitely result in voltage instability for the system if the operation of the system utility not able to maintain the voltage within the allowable limits. Keeping the voltage within s specific allowable limits is essential to provide the best service quality for the customers [5].

The risk of maintaining the buses required voltage within the allowable limits and so keeping the system stable is a challenge for those power system networks that operated under highly stressed conditions due to its limited ability to expand the transmission system [6].

At particular points in power systems, Flexible Alternating Current Transmission Systems (FACTS) can be installed to provide adjustment capabilities for the altering voltage, phase angle and/or impedance. FACTS device are innovative new technology which provide a method to ad control ability to the power system network. On other hand, supporting the voltage during sudden events can be provided by Static Var Compensator (SVC) which behaves as a fast acting dynamic reactive compensation [7].

Effective and fast control over the electrical systems' parameters can be achieved by using FACTS technology. In FACTS, with the help of computerized solutions and the usage of the latest advances in power electronics and physics, FACTS considered as the most valuable and popular compensation technique in power systems applications. In addition to its popularity among the other compensation techniques, FATCTS are essential device in the modern power systems where it provides more flexibility and controllability in the power flow along the transmission lines [8] [9].

In power systems, the bus voltage affected in case of any mismatch in the balance of the 
generated and absorbed reactive power. Keeping that balance is essential to maintain the voltage of the bus within the allowable limits. With SVCs characteristics, over wide range of loads, an accurate voltage control can be applied on the buses. SVCs are provide a step less and soft variation of reactive power that can feed the buses and hence the transmission lines [10]. In transmission network, the power transfer capability and the power stability as well could be effectively enhanced by using the Static VAR compensator (SVC) which is one of the promising solutions, cost effective and best for improving the power factor by compensating the reactive power. [11][12]. Many features may archived by using the SVCs. When the reactive power is monitored and controlled on a certain point in the system, voltage breakdown could be prevented in addition to enhancing the power factor of the systems. Moreover, the transient stability of the system has proven to be improved by using the SVCs [13]. Compensating the instable voltage may occur in two cases; either decreasing or increasing the voltage level of the system in both cases the SVCs are used to regulate the transmission voltage by connecting them to the power system in specific points. Thyrsitor controlled reactors are activated by the SVC to consume VARs from the system in order to decrease the system voltage in case of leading (capacitive) load. On other hand, capacitor banks are activated and used to increase the voltage level of the system in case of lagging (inductive) load conditions [14].

Identifying the optimal location for the SVCs and other types of FACTS is an importance factor to practically improve the voltage stability fi the power system. In addition, Direct and flexible control of power transmission can be achieved by utilizing FACTS devices which are enhance the performance of the power system and hence the system stability [15]. Direct and flexible control of power transmission can be achieved by utilizing FACTS devices which are enhance the performance of the power system and hence the system stability.

The SVC is basically used to regulate the transmission voltage and providing a damping effect over the power swings by controlling the reactive power. Controlling the reactive power in optimum way leads to a significant power loses reduction [1]. If the voltage of the system intended to be kept within a specified allowable range, a continuous adjustment of the reactive power must be made through the SVC [16].

\section{SVC COMPENSATOR}

SVCs is acting as reactive power generators or loads when they are connected in shunt at the buses of electrical networks, where practically they are applied to rapidly control the voltage of the desired weak bus in the transmission section of the network [17].

Figure 1 shows the equivalent circuit of the SVC, while Figure 2 illustrates the detailed diagram of a standard configuration of SVC. SVC in Figure 2 is specified to be ThyristorControlled-Reactor Fixed Capacitor (TCR-FC) which is power electronics and electronics components for harmonic filtering purposes, and coupling transformer [18].

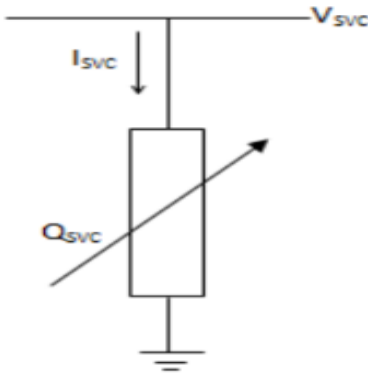

Figure 1: SVC Equivalent circuit diagram [19] 


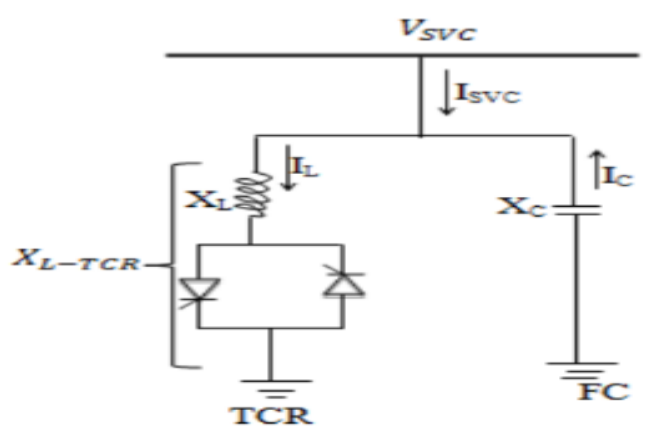

Figure 2: TCR-FC SVC Configuration [19]

\section{CASE STUDY}

$132 \mathrm{KV}$, 30-bus power systems in Kurdistan region, Iraq, were used as a case study in this paper. It consists of 30 buses, 5 generation stations, and $42 \mathrm{HV}$ transmission lines.

\subsection{The System Simulated Model}

The considered system under this study has been modeled by using MATLAB/SIMULINK application program, considering that the voltage sources are representing the generation station and the combination of the resistance-reactance is the equivalent to the transmission lines as shown in Figure 3.

\subsection{Bus Mathematical Analysis}

The transmission line between any two given buses in Figure 3 could be modeled as shown in Figure 4, including resistance-reactance combination from bus $i$ to bus $k$.

Where;

$V_{i}=$ complex voltage at bus $\mathrm{i}$

$V_{k}=$ complex voltage at bus $\mathrm{k}$

$R_{i k}=$ resistance for any given transmission

$X_{i k}=$ reactance for any given transmission

$I_{i k}=$ complex current flowing from bus $i$ to $k$

$Z_{i k}=$ impedance for any given transmission line

From Figure 4, the voltage drop between buses $i$ and $k$ given as

$V_{d}=V_{i}-V_{k}$

According to Ohm's law, the complex current flowing from bus $i$ to bus $k$ is expressed as:

$I_{i}=\frac{V_{i}-V_{K}}{Z_{i K}}$

$Z_{i K}=R_{i K+} X_{i K}$

An admittance form of eq. (2) can be written as:

$I_{i}=\left(V_{i}-V_{K}\right) y_{i k}$

Where: $y_{i k}=1 / Z_{i K}$ and is defined as the admittance of the transmission line.

Refering to [20], the complex power $S_{i K}$ is expresses as:

$S_{i K}=V_{i} * I_{i K}^{*}$ 
With respect to real and reactive power, $P$ and $Q$ respectively, eq. (4) re-written as: $S_{i K}=P_{i K}+Q_{i K}=V_{i} * I_{i K}{ }^{*}$

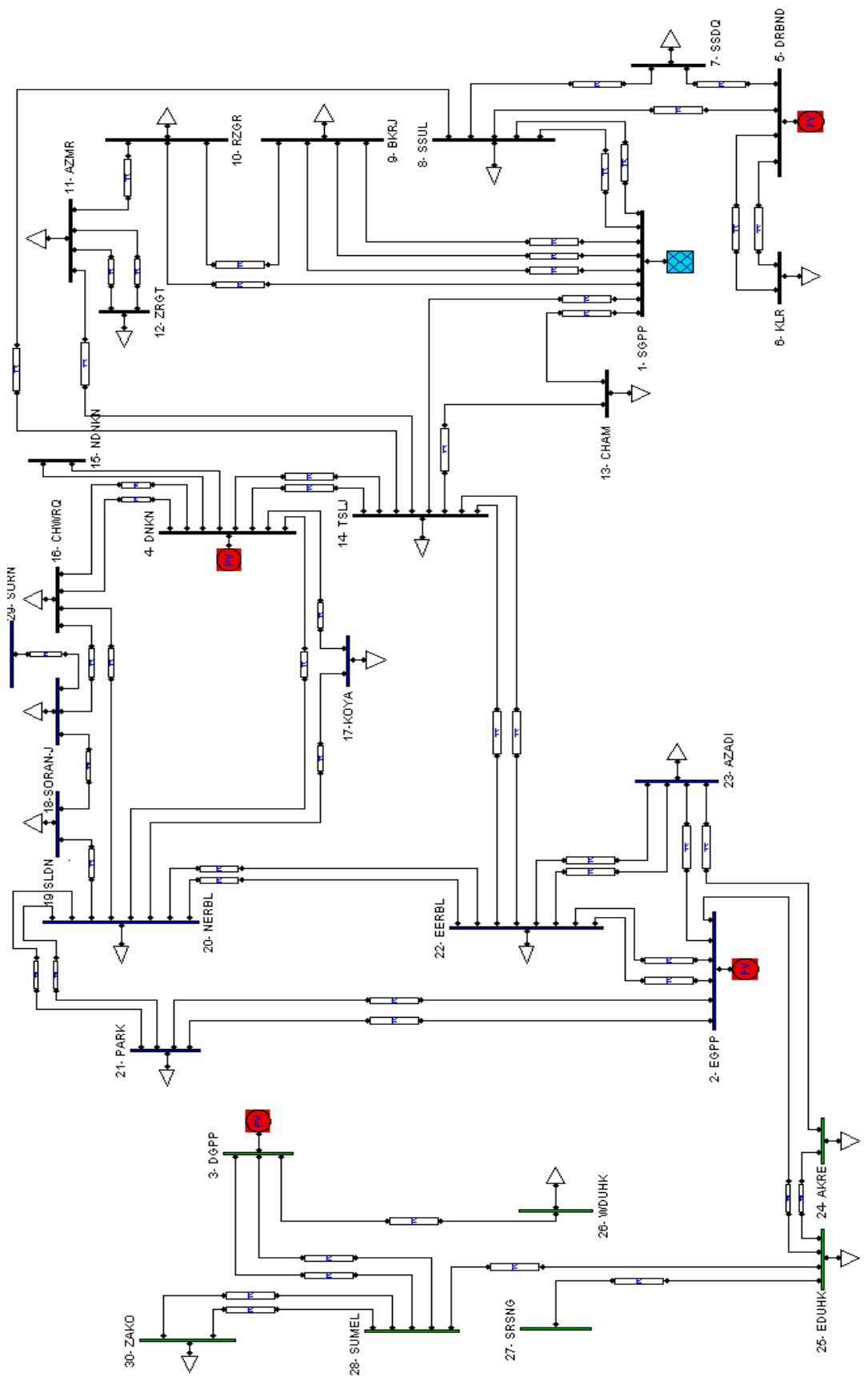

Figure 3: KRG 30-bus power system. 


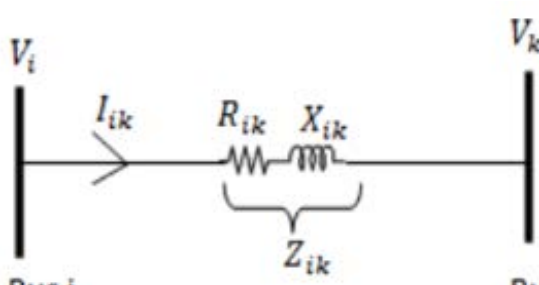

Bus i

Bus $k$

Figure 4: Transmission Line Model.

\subsection{Indexes based Voltage Instability measure}

The voltage sensitivity factor is represented by the absolute ratio of the per unit voltage change $d V_{i}$ to the total active power change $d P_{\text {total }}$ for the $i^{\text {th }}$ bus in the power system of the study case, where it could be written as $\left|d V_{i} / d P_{\text {total }}\right|$. Knowing that the total active load change for any given bus is the same for all other buses in the same network, therefore the voltage stability factor can be considered as an index of the differential change of the voltage for the given $i^{\text {th }}$ bus [21].

The $i^{\text {th }}$ bus that gives an index value $\left(d V_{i}\right)$ closest to 1 , that $i^{\text {th }}$ bus is considered as a critical bus and may lead to instability in the system. As a result, the critical $i^{\text {th }}$ bus is the weakest bus which is nominated to be the optimal location for placing the SVCs [22].

\section{RESULTS AND DISCUSSION}

\subsection{Voltage sensitivity factor of buses}

Table (1) and Figure 5 are explaining the voltage sensitivity factors, $|d V i / d P t o t a l|$ for all buses in the network of the study case considering buses 1 to 5 as generation buses. Bus-29, Bus-18 and Bus-12 have higher voltage sensitivity factor than other buses. In addition, referring to the voltage sensitivity factor criteria, it can be clearly seen that Bus-15 is most strong bus among the other load buses.

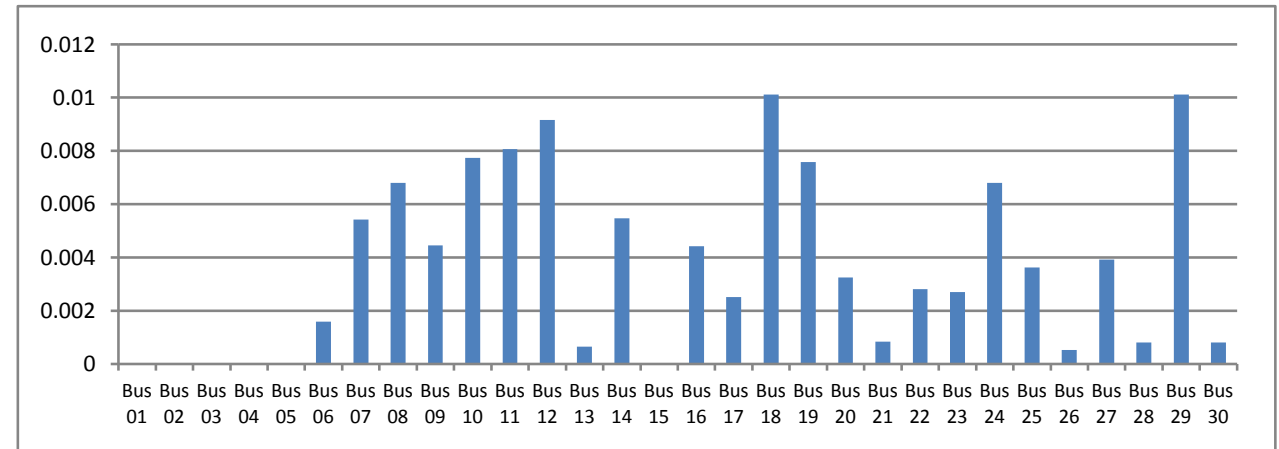

Figure 5: Voltage Sensitivity Factors of buses.

Table1: Voltage Sensitivity Factors of buses.

\begin{tabular}{cc}
\hline Bus No. & Voltage Sensitivity Factor \\
\hline $\mathbf{0 1}$ & 0 \\
\hline $\mathbf{0 2}$ & 0 \\
\hline $\mathbf{0 3}$ & 0 \\
\hline $\mathbf{0 4}$ & 0 \\
\hline $\mathbf{0 5}$ & 0 \\
\hline $\mathbf{0 6}$ & 0.001587 \\
\hline $\mathbf{0 7}$ & 0.005427 \\
\hline $\mathbf{0 8}$ & 0.006792 \\
\hline $\mathbf{0 9}$ & 0.004445 \\
\hline $\mathbf{1 0}$ & 0.007743 \\
\hline $\mathbf{1 1}$ & 0.008068 \\
\hline
\end{tabular}




\begin{tabular}{lc}
\hline $\mathbf{1 2}$ & 0.009164 \\
\hline $\mathbf{1 3}$ & 0.000646 \\
\hline $\mathbf{1 4}$ & 0.00547 \\
\hline $\mathbf{1 6}$ & 0.0000126 \\
\hline $\mathbf{1 7}$ & 0.004429 \\
\hline $\mathbf{1 8}$ & 0.00251 \\
\hline $\mathbf{1 9}$ & 0.010118 \\
\hline $\mathbf{2 0}$ & 0.007587 \\
\hline $\mathbf{2 1}$ & 0.003248 \\
\hline $\mathbf{2 3}$ & 0.000842 \\
\hline $\mathbf{2 4}$ & 0.002809 \\
\hline $\mathbf{2 5}$ & 0.002696 \\
\hline $\mathbf{2 6}$ & 0.006795 \\
\hline $\mathbf{2 7}$ & 0.003617 \\
\hline $\mathbf{2 8}$ & 0.000522 \\
\hline $\mathbf{2 9}$ & 0.003924 \\
\hline $\mathbf{3 0}$ & 0.00081 \\
\hline & 0.01012 \\
\hline
\end{tabular}

\subsection{SVCs Placing}

In order to improve the voltage stability margin in the network, the optimal locations for placing the SVCs are the weakest buses that identified previously from Table (1) or Figure 5. Where Bus-29, Bus-18 and Bus-12 have the highest voltage sensitivity factors from the most to the least critical bus respectively, which are selected to place the SVCs

As its clear in Table (2), the results of SVCs placing on the weakest buses result in an improvement in voltage profile of all buses, the percent increase in bus voltages of buses 10 , 11 and 12 are $13.19 \%, 15.29 \%$, and $16.17 \%$ respectively. Furthermore, percent decrease of total losses for the active and reactive power in the network is $14.81 \%, 5.26 \%$ respectively. Figures (6), (7) and (8) indicate buses 10, 11 and 12 voltage profiles respectively for base case and after SVCs placing. Without SVCs, the system reaches its steady state stability limit with load scaling factor of 3.6387, while with SVCs the scaling factor increases to be 3.6562; i.e. the percent increase in loading factor is $0.47 \%$.

Table 2: Bus voltages for base case and after SVCs placing.

\begin{tabular}{ccc}
\hline \multirow{2}{*}{ Bus No. } & \multicolumn{2}{c}{ Bus Voltage } \\
\cline { 2 - 3 } & Before SVCs Placing & After SVCs Placing \\
\hline $\mathbf{0 1}$ & 1 & 1 \\
\hline $\mathbf{0 2}$ & 1 & 1 \\
\hline $\mathbf{0 3}$ & 1 & 1 \\
\hline $\mathbf{0 4}$ & 0.984 & 0.984 \\
\hline $\mathbf{0 5}$ & 0.982 & 0.982 \\
\hline $\mathbf{0 6}$ & 0.882275 & 0.881722 \\
\hline $\mathbf{0 7}$ & 0.722914 & 0.774475 \\
\hline $\mathbf{0 8}$ & 0.676491 & 0.681983 \\
\hline $\mathbf{0 9}$ & 0.787712 & 0.789812 \\
\hline $\mathbf{1 0}$ & 0.62813 & 0.711025 \\
\hline $\mathbf{1 1}$ & 0.60914 & 0.702292 \\
\hline $\mathbf{1 2}$ & 0.604213 & 0.701937 \\
\hline $\mathbf{1 3}$ & 0.968738 & 0.973639 \\
\hline $\mathbf{1 4}$ & 0.733361 & 0.784146 \\
\hline $\mathbf{1 5}$ & 0.979250 & 0.979252 \\
\hline $\mathbf{1 6}$ & 0.768444 & 0.773911 \\
\hline $\mathbf{1 7}$ & 0.858702 & 0.861487 \\
\hline $\mathbf{1 8}$ & 0.503827 & 0.519718 \\
\hline $\mathbf{1 9}$ & 0.628595 & 0.639851 \\
\hline $\mathbf{2 0}$ & 0.83803 & 0.843209 \\
\hline $\mathbf{2 1}$ & 0.957981 & 0.959062 \\
\hline
\end{tabular}




\begin{tabular}{ccc}
\hline $\mathbf{2 2}$ & 0.859937 & 0.865655 \\
\hline $\mathbf{2 3}$ & 0.86508 & 0.869851 \\
\hline $\mathbf{2 4}$ & 0.670592 & 0.670704 \\
\hline $\mathbf{2 6}$ & 0.826197 & 0.824839 \\
\hline $\mathbf{2 7}$ & 0.970329 & 0.970165 \\
\hline $\mathbf{2 8}$ & 0.809639 & 0.808161 \\
\hline $\mathbf{2 9}$ & 0.962176 & 0.961844 \\
\hline $\mathbf{3 0}$ & 0.50391 & 0.519803 \\
\hline Active Power looses & 0.963204 & 0.962872 \\
\hline Reactive Power losses & 9.113798 & 7.763851 \\
\hline Loading Factor & 41.42701 & 39.24775 \\
\hline
\end{tabular}

(6) after

From figure

insertion SVC the voltage profile improved from 0.62813 p.u. to 0.711025 p.u., From figure (7) after insertion SVC the voltage profile improved from 0.60914 p.u. to 0.702292 p.u. and From figure (8) after insertion SVC the voltage profile improved from 0.604213 p.u. to 0.701937 p.u.
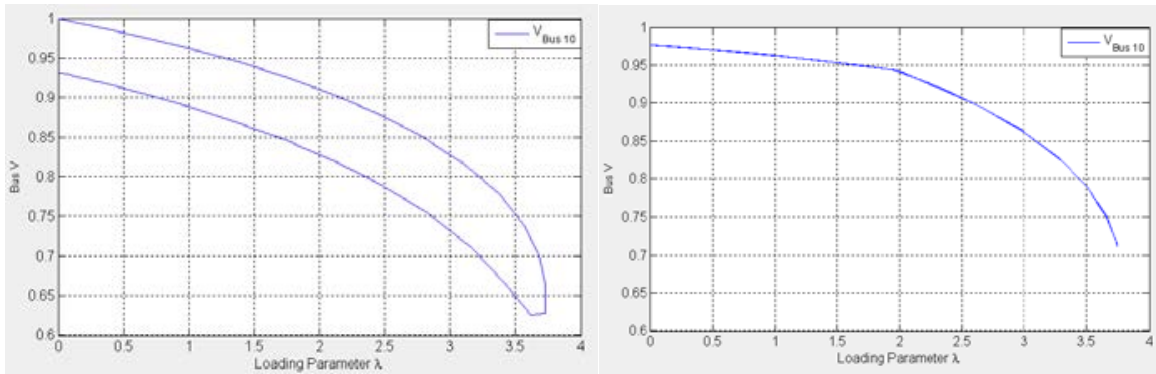

Figure 6: Voltage profile of Bus -10 before and after insertion of SVC.
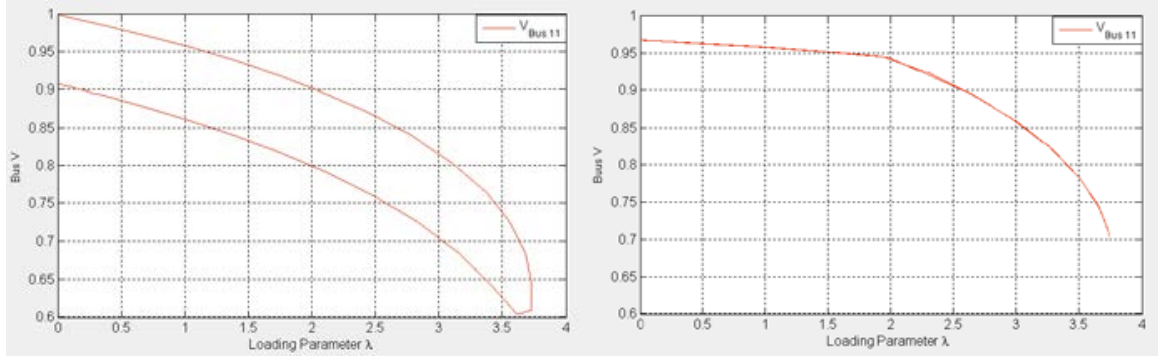

Figure 7: Voltage profile of Bus -11 before and after insertion of SVC.
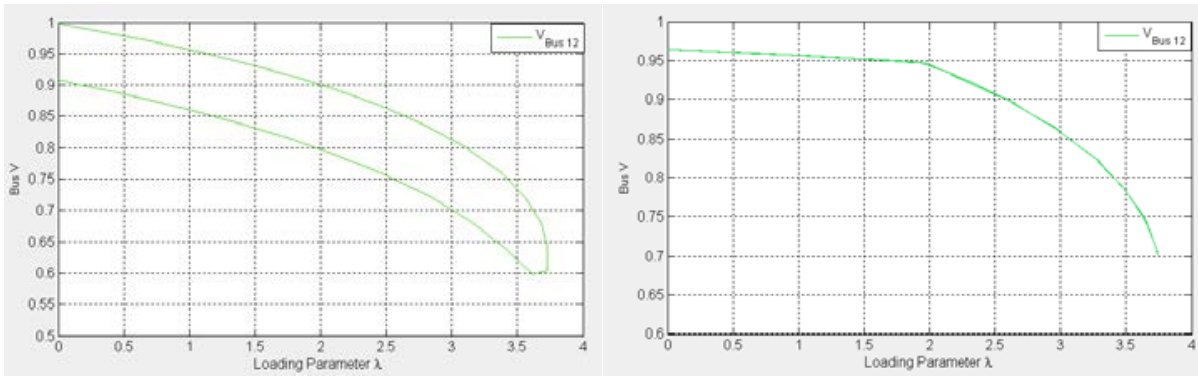

Figure 8: Voltage profile of Bus -12 before and after insertion of SVC.

\section{CONCLUSION}

In this research, the optimal buses for placing SVCs are identified by using the voltage sensitivity factor determination criteria. Placing SVCs at the weakest buses result in notable improvements in the voltage stability limit and a clear reduction of the active and reactive power losses in the system. The increment of the loading factor after placing the SVCs in the optimal buses that identified had proved that the voltage stability is enhanced. Also, it can be 
conclude that buses that have better voltage characteristics than other buses are the buses that have smaller sensitivity factor which are either close to the generating units or light loaded. It could be clearly seen that the average change in voltage profile for the system results in about $2.3 \%$ in per unit change for the buses which is provide the system with a good improvement in stability.

\section{REFERENCE}

[1] S. Raja, A. Anandhi, "Voltage Stability Improvement by static TATIC VAR Compensator", International Journal of Science, Engineering and Technology Research (IJSETR), Volume 4, Issue 5, May 2015.

[2] I. Monirul, M. Nadarajah, H. Jahangir, S. Rakibuzzaman, "Dynamic voltage stability of unbalanced distribution system with high penetration of single-phase PV units", The Journal of Engineering. Vol. 2019, No.17, pp. 4074-4080, 2019

[3] M. Yao, D. K. Molzahn and J. L. Mathieu, “An Optimal Power-Flow Approach to Improve Power System Voltage Stability Using Demand Response,” IEEE Transactions on Control of Network Systems, vol. 6, no. 3, $\begin{array}{llll}\text { pp. 1015-1025, } & \text { Sept. }\end{array}$ doi: 10.1109/TCNS.2019.2910455

[4] N. K. Sharma, A. N. Tiwari, "Prevention of Voltage Instability by Using FACTS Controllers in Power Systems: A Literature Survey”, International Journal of Engineering Science and Technology, Vol. 2(5), pp 980-992, 2010.

[5] N. Natasha, M. Sujata, "Voltage Collapse: Causes and Prevention”, International Journal of Engineering Research \& Technology (IJERT), Vol. 4, No. 2, 2016.

[6] B. Singh, "Applications of FACTs Controllers in Power Systems for Enhance the Power System Stability: A State-of-the-Art”, International Journal of Reviews in Computing, Vol. 6, 2011.

[7] N. Mark Ndubuka, "Voltage Stability Improvement using Static Var Compensator in Power Systems", Leonardo Journal of Sciences, Issue 14, p. 167-172, January-June 2009.

[8] K. Naseeb, S. Sajida, "A Survey on Different types of Flexible AC Transmission Systems (FACTS) Controllers”, IJEDR | Volume 5, Issue 4, 2017.

[9] K. Rajesh, G. Prashant, Y. Balram, ”S Survey Paper on IEEE 14-bus System with FACTS Technology”, International Journal of Innovative Research in Computer and Communication Engineering, Vol. 5, Issue 10, October 2017.

[10] P. Manan, J. G. Jamnani, “Closed Loop Control and Harmonic Analysis of SVS for Maintaining Voltage Profile of Power System” International Journal of Engineering Research and Development (IJERD) Recent trends in Electrical and Electronics \& Communication Engineering (RTEECE 17th - 18th April 2015).

[11] S. Sita, H. Jitendra, K. Ashutosh, “Application of SVC on IEEE 6 Bus System for Optimization of Voltage Stability”, Indonesian Journal of Electrical Engineering and Informatics (IJEEI) Vol. 3, No. 1, March 2015.

[12] G. Vijay S., C. Dinesh M., "Power Quality Improvement By Svc Using PIC18F4", International Journal for Research in Applied Science \& Engineering Technology (IJRASET), Vol. 5, No. VI, 2017.

[13] S. Reza, I. Khodakhast, H. B. Mohammad, A. M. Navid, "Enhancement of Power System Dynamic Performance using Coordinated Control of FACTS Devices”, Indian Journal of Science and Technology, Vol.7, No.10, pp. 1513-1524, 2014.

[14] P. Kaibalya, S. Soumyakanta, R. Sreyasee, "Prototype design of power factor correction circuit for transmission lines using Thyristor switched capacitor scheme”, World Journal of Modelling and Simulation, Vol. 13, No. 4, pp. 314-321, 2017.

[15] L. Abdelaziz, B. Mohamed, “Optimal Location of SVC for Voltage Security Enhancement using MOPSO”, Third International Conference on Electrical Engineering-ICEE'09, 19-21 May, 2009.

[16] A. Pabla, Electric power distribution. New York: McGraw-Hill, 2005.

[17] A. Banga, S. S. Kaushik, "Modeling and Simulation of SVC Controller for Enhancement of Power System Stability,” International Journal of Advances in Engineering and Technology, Vol. 1, pp.79-84, 2011.

[18] M. M. Biswas, K. D. Kamol, "Voltage Level Improving by Using Static VAR Compensator," Global Journal of Researches in Engineering, Vol.11, pp.12-18, 2011.

[19] M. Nwohu, "Voltage Stability Improvement using Static Var Compensator in Power Systems”, Leonardo Journal of Sciences, Issue 14, pp. 167-17, 2009.

[20] A. J. Conejo, 2011, Load Flow Lecture, University of Castilla - La Mancha.

[21] B. Kessk-N Mehemet, “Continuation Power Flow and Voltage Stability in Power Systems”, Master of Science Thesis, Middle East Technical University, September, 2007.

[22] A. Ramasamy, R. Verayiah, H. I. Zainal Abidin, I. Musirin, A. A. Rahim, "Performance of Fast Voltage Stability Index (FVSI) as an indicator for Under Voltage Load Shedding Scheme in a Bulk Power System Network”, International Journal of Power, Energy and Artificial Intelligence, No.1, pp 157-165, 2009. 